\title{
Effects of sterigmatocystin on esophageal epithelium and experimental reflux esophagitis in rats
}

\author{
PAN-ZHUO TONG ${ }^{1,2}$, GUO-JIAN ZHANG $^{3}$, XIANG-HONG ZHANG $^{1}$, XIA YAN $^{1}$ and JUN-LING WANG ${ }^{1}$ \\ ${ }^{1}$ Department of Pathology, Second Hospital of Hebei Medical University, Shijiazhuang, Hebei 050000; Departments of \\ ${ }^{2}$ Dermatology and ${ }^{3}$ Surgery, Second Hospital of Hebei Medical University, Shijiazhuang, Hebei 050017, P.R. China
}

Received February 19, 2013; Accepted August 2, 2013

DOI: $10.3892 / \mathrm{mmr} .2013 .1631$

\begin{abstract}
Patients with reflux esophagitis experience an increased incidence of esophageal cancer. In China, this may be the result of contamination of the food supply by Aspergillus fungi, which is known to harbor sterigmatocystin, a carcinogenic mycotoxin. To delineate the potential link between sterigmatocystin and esophageal cancer, an experimental model of reflux esophagitis was developed in rats that had undergone a cardiectomy and partial pylorus ligation. The rats were treated with sterigmatocystin or saline, and esophageal squamous cell hyperplasia was assessed based on the pathological evaluation. The expression of proliferating cell nuclear antigen (PCNA), transporter associated with antigen processing 1 (TAP1) and low molecular weight protein 2 (LMP2) was determined by immunohistochemistry. Intraperitoneal administration of sterigmatocystin promoted the proliferation of squamous epithelium. In addition, it also increased the expression of PCNA in esophageal epithelial cells in rats with reflux esophagitis and was correlated with the increased severity of epithelial hyperplasia. The expression levels of TAP1 and LMP2, which are located in the cytoplasm of esophageal epithelial cells, were reduced in rats with reflux esophagitis, and sterigmatocystin exposure further decreased the expression. Thus, the downregulation of TAP1 and LMP2 proteins by sterigmatocystin may directly affect tumor immunity by allowing transformed cells to escape the host immune surveillance, thereby promoting esophageal cancer.
\end{abstract}

Correspondence to: Dr Xiang-Hong Zhang, Department of Pathology, Second Hospital of Hebei Medical University, No. 215 Heping Xilu, Shijiazhuang, Hebei 050000, P.R. China

E-mail: zhangxianghong2008@163.com

Key words: sterigmatocystin, reflux esophagitis, esophageal squamous epithelial cell, proliferating cell nuclear antigen, transporter associated with antigen processing 1, low molecular weight protein 2

\section{Introduction}

Sterigmatocystin is a mycotoxin derived from the Aspergillus fungus, and is a known carcinogen (1-4). The development of esophageal cancer has been linked to sterigmatocystin consumption $(1,3)$; however, a variety of factors, including esophageal mucosal injury and immune function, are likely to be involved in disease onset $(5,6)$. Epidemiological studies have demonstrated that the eating habits of residents in China contribute to the relatively high incidence of esophageal cancer and the prevalence of reflux esophagitis $(6,7)$. The presence of contaminating mycotoxins in the Chinese food supply is a serious and widely recognized issue $(1,8)$. Previous studies of the sterigmatocystin mycotoxin have demonstrated its ability to negatively impact immune function. Sterigmatocystin treatment of human peripheral blood mononuclear cells resulted in a significant downregulation of the gene expression of transporter associated with antigen processing 1 (TAP1) and low molecular weight protein 2 (LMP2), two key regulators of the immune response. Moreover, sterigmatocystin was observed to inhibit the expression of human leukocyte antigen (HLA)-I in human esophageal squamous cells in a dose-dependent manner $(9,10)$. Considering the extent of sterigmatocystin mycotoxin contamination in the general food supply and its suggested link to esophageal cancer and decreased immune function, this study was conducted to investigate the effects of sterigmatocystin exposure in rats with reflux esophagitis. Proliferating cell nuclear antigen (PCNA), LMP2 and TAP1 protein expression was determined in these rats in the absence and presence of sterigmatocystin.

\section{Materials and methods}

Materials. Thirty healthy male Wistar rats (age, 4 weeks; weight, 40-50 g) were purchased from the Experimental Animal Center of Hebei Medical University in China (Hebei, China). This study was conducted with the approval of the Local Ethics Committee of Hebei Medical University (no. DK051207). Sterigmatocystin was purchased from Sigma-Aldrich (St. Louis, MO, USA). Goat anti-human LMP2 polyclonal antibody and goat anti-human TAP1 polyclonal antibody were purchased from Santa Cruz Biotechnology, Inc. (Santa Cruz, CA, USA). The PCNA polyclonal antibody was purchased from Wuhan Boster Biological Technology, 
Ltd. (Wuhan, China). The immunohistochemical staining kit (model no. 1386323) was purchased from Beijing Zhong Shan Golden Bridge Biotechnology Co., Ltd. (Beijing, China).

Grouping and modeling. Rats $(\mathrm{n}=30)$ were randomly divided into the following three groups $(n=10)$ : i) Control group, treated with placebo and surgery; ii) esophagitis model group, experimental reflux esophagitis achieved by cardiectomy and ligation of half of the pylorus without sterigmatocystin; and iii) sterigmatocystin-treatment group, the reflux esophagitis model with sterigmatocystin treatment. Surgery was performed when the rats acclimated to their environment for 1-2 days.

In the control group (placebo treatment), esophageal diameter was small $(\sim 3 \mathrm{~mm})$, thus the surgery required precise technical procedures. While under $10 \%$ chloral hydrate anesthesia ( $3 \mathrm{ml} / \mathrm{kg}$, administered intraperitoneally), the abdominal cavity was opened to expose the stomach and esophagus, the anterior branch of the left gastric artery was ligated to reduce bleeding and the abdomen was closed. Prior to and following surgery, rats were allowed liquid glucose only for $24 \mathrm{~h}$. Additionally, a liquid diet was provided for three days following surgery, subsequent to which a normal diet was resumed. After 7 days, placebo treatment (intraperitoneal injection with saline) was administered once a day for seven days.

In the esophagitis model group, the abdominal cavity was opened to expose the stomach and esophagus while mice were under anesthesia (10\% chloral hydrate administered intraperitoneally). The anterior branch of the left gastric artery was ligated and the sphincter was cut off by the removal of the cardiac smooth muscle; simultaneously, one-third of the circumference of the lower esophageal smooth muscle was removed. The abdomen was closed and the rats were provided with the same diet and saline injections as described for the control group.

In the sterigmatocystin treatment group, anesthesia, surgical procedures and food intake were identical to those described for the experimental esophagitis group. Seven days after the normal diet was resumed, an intraperitoneal injection of sterigmatocystin $(30 \mu \mathrm{g} / \mathrm{kg})$ was administered, followed by consecutive daily sterigmatocystin injections for seven days. A parenteral route of sterigmatocystin administration was performed rather than peroral administration, as this route failed to induce esophageal cancer in our previous study (1).

A number of rats from all groups were sacrificed 5 or 11 weeks after the seventh day of treatment $(n=6$ in the control group, $n=8$ in the esophagitis model group, $n=10$ in the sterigmatocystin treatment group). The groups were split evenly into two groups for the two sacrifice time points.

Pathological analysis. The esophagus was excised en bloc and examined for general morphological changes. Tissue samples were then placed in formalin solution to be longitudinally fixed. Conventional hematoxylin and eosin (H\&E) staining was performed on the sections and histological changes were evaluated by light microscopy.

Immunohistochemical detection of PCNA, TAP1 and LMP2 expression. Immunohistochemical staining was performed according to the manufacturer's instructions (Santa Cruz Biotechnology, Inc., Santa Cruz, CA, USA). Phosphate-buffered saline was used in lieu of primary antibody as a negative control.

Criteria for pathological analysis. Two blinded experts performed the pathology-based diagnosis. The esophagitis classification of specimens was conducted according to the following Tokyo standards $(11,12)$ : 0 , no esophagitis observed; 1 , redness or white turbidity; 2 , erosion, ulcer esophageal length of $\leq 1 / 3$ of the total oesophageal length (units) and non-integration; 3, erosion, esophageal ulcers account for the full length (1/3-2/3 units) and with integration; and 4 , erosion, esophageal ulcers account for the full length ( $>2 / 3$ units). Inflammation scores were allocated based on the following criteria (per high power field): 0 , no inflammatory cell infiltration; 1 , a small number of inflammatory cells; 2, moderate inflammatory cell infiltration; and 3, a large quantity of inflammatory cell infiltration. Hyperplasia scores were allocated according to the following classifications: 0, normal esophageal stratified squamous epithelium basal cell layer; 1 , basal cell hyperplasia was $1 / 3$ of the total thickness; and 2, basal cell hyperplasia was $1 / 3-2 / 3$ of the total thickness.

Criteria for immunohistochemical analysis. This was performed by two common film reading statistical procedures. For PCNA, the number of positive cells per high power field (x400) for 10 fields was counted. The average rate of positive cells for every 100 epithelial cells was used as the PCNA labeling index (PCNA LI). For TAP1 and LMP2, the number of positive cells per high power field (x400) for 10 fields was counted. The average rate of positive cells for every 100 epithelial cells was used as the rate of expression of TAP1 and LMP2.

Statistical analysis. SPSS 11.0 (SPSS Inc., Chicago, IL, USA) statistical software was used for single-factor analysis of variance (ANOVA) and Student-Newman-Keuls test was used for pairwise comparisons. $\mathrm{P}<0.05$ was considered to indicate statistically significant differences.

\section{Results}

Condition of rats. One rat died of an anesthesia overdose during the surgical procedure, while a second rat died due to an esophageal perforation. Following surgery, two rats died as a result of intestinal obstruction and an additional two rats died from unknown causes. A total of 24 rats survived and completed the study. Reflux behavior via the mouth was observed in the esophagitis model and sterigmatocystin-treated animals after 1 week, and suggested that the reflux surgery had been successful. Compared with the control and esophagitis model groups, the sterigmatocystin-treated animals exhibited a reduced intake of food and water, reduced locomotor activity and a less glossy coat.

Esophageal specimens from rats with reflux esophagitis. Smooth and normal esophageal mucosa were identified in the control group (Fig. 1). By contrast, the esophagitis model and sterigmatocystin-treated groups exhibited mucosal congestion with edema, erosion and ulcers (Fig. 2). 
Table I. Esophageal squamous cell score in reflux esophagitis rats.

\begin{tabular}{|c|c|c|c|}
\hline Score & $\begin{array}{l}\text { Control group } \\
\qquad(\mathrm{n}=6)\end{array}$ & $\begin{array}{l}\text { Esophagitis model group } \\
\qquad(\mathrm{n}=8)\end{array}$ & $\begin{array}{l}\text { ST-treated group } \\
\qquad(\mathrm{n}=10)\end{array}$ \\
\hline Inflammation score at week 5 & & $1.47 \pm 0.46$ & $1.89 \pm 0.10$ \\
\hline Inflammation score at week 11 & & $1.70 \pm 0.28$ & $1.87 \pm 0.447$ \\
\hline Hyperplasia severity score at week 5 & & $1.43 \pm 0.06$ & $1.68 \pm 0.08^{\mathrm{a}}$ \\
\hline Hyperplasia severity score at week 11 & & $1.40 \pm 0.15$ & $1.62 \pm 0.14^{\mathrm{a}}$ \\
\hline PCNA LI & $21.50 \pm 9.79$ & $49.17 \pm 18.67$ & $70.10 \pm 12.55^{\mathrm{a}}$ \\
\hline
\end{tabular}

${ }^{a} \mathrm{P}<0.05$ vs. esophagitis model group. ST, sterigmatocystin; PCNA LI, proliferating cell nuclear antigen labeling index.

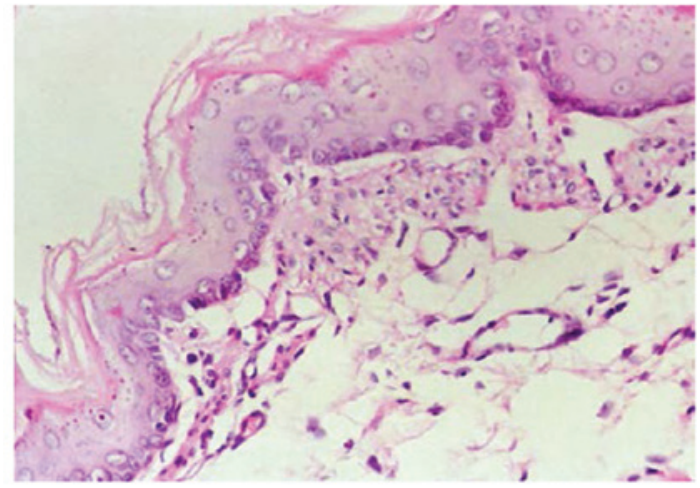

Figure 1. Esophageal squmaous epithelium of rats in the control group (stain, heamatoxylin and eosin; magnification, $\mathrm{x} 400$ )

$H \& E$ staining of the esophagus in rats with reflux esophagitis. At 5 weeks following treatment, the esophageal tissues in the esophagitis model group exhibited epithelial hyperplasia, acanthosis of the basal layer in association with general congestion and edema, vein dilation, angiogenesis, submucosal infiltration of inflammatory cells and the presence of eosinophils, neutrophils and lymphocytes. In severe cases, the esophagus presented mucosal cell degeneration and necrosis with epithelial shedding and granulation tissue formation. An ulcer structure was identified in one case. The esophagus in sterigmatocystin-treated rats showed esophageal epithelial hyperplasia, acanthosis of the basal layer and the majority of the nuclei exhibited loss of polarity, mild to moderate atypia, congestive edema, vein dilation and angiogenesis. In addition, mucous membranes exhibited inflammatory cell infiltration consisting predominantly of neutrophils and lymphocytes, severe mucosal cell degeneration and necrosis and a typical ulcer detached structure; however, no atypical cells were observed in the groups.

At 11 weeks, the esophagus in the esophagitis model and sterigmatocystin-treated groups exhibited no ulcers, but did show esophageal mucosal epithelial hyperplasia, mucosal congestion and edema and cystic fibrosis in the submucosa. In addition, no atypical cells were observed in the groups.

At weeks 5 and 11, the sterigmatocystin-treated group had marginally higher inflammation scores compared with the esophagitis model group; however, the difference was not statistically significant (Table I; $\mathrm{P}>0.05$ ).
A

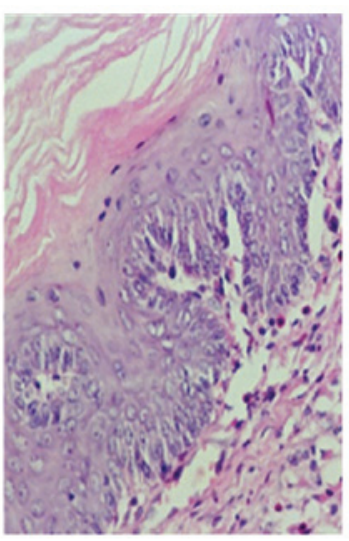

C
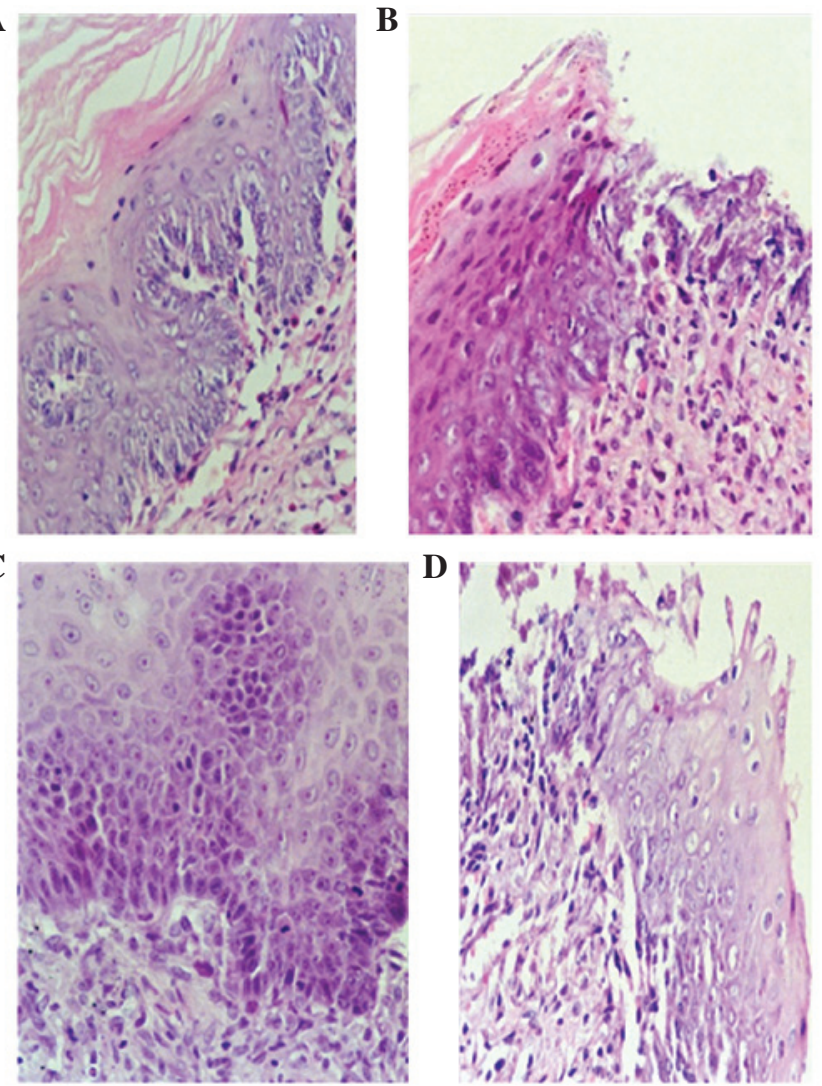

D

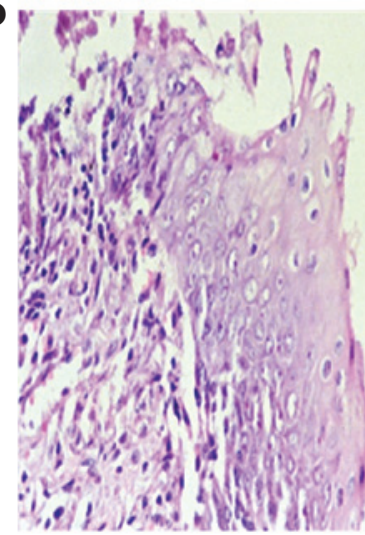

Figure 2. Pathological changes in the esophageal squamous epithelium in the esophagitis model and sterigmatocystin-treated groups. The inflammation score was not significantly different (stain, heamatoxylin and eosin; magnification, $\mathrm{x} 400$ ). (A) Esophagitis model group, inflammation score at week 5. (B) Esophagitis model group, ulcers at week 5. (C) Sterigmatocystin-treated group, inflammation score at week 5. (D) Sterigmatocystin-treated group, ulcers at week 5 .

The hyperplasia severity score (Table I) for the sterigmatocystin-treated group was higher than that of the model group at weeks 5 and 11. Pairwise analysis between the sterigmatocystin-treated and esophagitis model groups indicated that the difference was statistically significant $(\mathrm{P}<0.05)$.

PCNA immunohistochemical analysis. PCNA-positive esophageal squamous cell nuclei were observed in rats with reflux esophagitis. This is depicted by brown-yellow granules in Fig. 3, which also represent cell proliferation. The prolifera- 
Table II. The rate of TAP1- and LMP2-positive cells identified by immunohistochemical staining.

\begin{tabular}{lll}
\hline Group & TAP1 (\%) & LMP2 (\%) \\
\hline Control & $71.17 \pm 6.62$ & $53.83 \pm 5.81$ \\
Esophagitis model & $36.17 \pm 5.31^{\mathrm{a}}$ & $34.50 \pm 6.44^{\mathrm{a}}$ \\
Sterigmatocystin-treated & $20.83 \pm 7.08^{\mathrm{a}}$ & $22.50 \pm 6.72^{\mathrm{a}}$ \\
\hline
\end{tabular}

${ }^{\mathrm{a}} \mathrm{P}<0.05$ vs. the control group. The number of animals used was 6, 8 and 10 for control, esophagitis and sterigmatocystin groups, respectively. TAP1, transporter associated with antigen processing 1; LMP2, low molecular weight protein 2.

tion index of PCNA expression was $21.50 \pm 9.79$ in the control group $(\mathrm{n}=6), 49.17 \pm 18.67$ in the esophagitis model group $(\mathrm{n}=8)$ and $70.10 \pm 12.55$ in the sterigmatocystin-treated group $(n=10)$ (Table I). According to statistical analysis using one-way ANOVA, the PCNA proliferation index was statistically different among the three groups $(\mathrm{P}<0.05)$. Further pairwise analysis of the control and model groups indicated that there was a significant difference between these two groups $(\mathrm{P}<0.05)$, and there was also a significant difference $(\mathrm{P}<0.05)$ between the model and sterigmatocystin-treated groups (Table I).

Thus, sterigmatocystin exacerbated reflux esophagitis with marked hyperplasia of the squamous epithelium. In addition, epithelial cells in the sterigmatocystin-treated group demonstrated significantly higher PCNA expression compared with that of the control and esophagitis model groups. It is suggested that gastric reflux increased the esophageal mucosal injury and promoted epithelial cell proliferation. Sterigmatocystin exposure resulted in more evident esophageal squamous cell hyperplasia.

TAP1 immunohistochemical analysis. TAP1 expression in the esophageal squamous cell cytoplasm was indicated by brown-yellow granules (Fig. 4). The rate of TAP1-positive cells in the esophagitis model $(n=8)$ and sterigmatocystin-treated groups $(\mathrm{n}=10)$ was reduced compared with that of the control group $(\mathrm{n}=6)(36.17 \pm 5.31$ and $20.83 \pm 7.08 \%$ vs. $71.17 \pm 6.62 \%$, respectively; P<0.051; Table II). TAP1 in the sterigmatocystin-treated group was significantly lower than that in the model group ( $\mathrm{P}<0.05$; Table II, Fig. 4).

LMP2 immunohistochemical analysis. LMP2 expression in the cytoplasm was indicated by brown-yellow granules (Fig. 4). The number of positive cells in the esophagitis model group $(n=8)$ and sterigmatocystin-treated group $(n=10)$ was lower than that in the control group $(\mathrm{n}=6)(34.50 \pm 6.44$ and $22.50 \pm 6.72 \%$ versus $53.83 \pm 5.81 \%$; $\mathrm{P}<0.05$, Table II). LMP2 expression in the sterigmatocystin-treated group was significantly lower than that in the model group $(\mathrm{P}<0.05$; Table II, Fig. 4).

\section{Discussion}

The fungus, sterigmatocystin, is a carcinogenic mycotoxin. Sterigmatocystin contamination of human food sources and animal feed has been confirmed in areas of China with a high incidence of esophageal cancer (1). Epidemiological studies have demonstrated that this high incidence of esophageal cancer in certain areas of China is also associated with a higher prevalence of reflux esophagitis $(6,7)$; thus, mycotoxin contamination is a serious public health concern. Zhang et al (1) demonstrated that sauerkraut soup samples and cornmeal bought from the Chinese Hebei Taihang Mountain region were significantly contaminated with sterigmatocystin. This study was extended by oral administration of purified sterigmatocystin to experimental animals, and it was determined that sterigmatocystin induced lung gland cancer and gastric epithelial dysplasia; however, not esophageal cancer. Thus, sterigmatocystin may cause esophageal cancer in conjunction with other underlying, yet unidentified, physiological and/or environmental factors.

Esophageal cancer is presumed to be the result of a multi-factorial process. The normal physiological function of the esophagus is a muscular tube through which food passes and esophageal squamous cells have the ability to repair themselves. However, esophageal mucosal injury appears to be an important factor involved in esophageal cancer onset in response to sterigmatocystin. Esophageal sphincter-relaxation may result in gastric reflux and esophageal inflammation. The rat gastro-esophageal junction area is similar to that in humans. Esophageal smooth muscle and gastric sling fibers of the gastroesophageal junction area are important anatomical structures controlling the anti-reflux mechanism $(13,14)$. Cardiac sphincter resection was successfully conducted in a rabbit model of reflux esophagitis by Xu et al (15). The esophagitis model and sterigmatocystin-treated groups presented with significant changes in esophagitis; however, the degree of inflammation was not significantly different between the two groups, suggesting a successful reflux esophagitis model in which there may be cardiac relaxation, reflux of gastric juice and injury to the esophagus $(14,16)$. There was noticeable esophageal squamous epithelium cell hyperplasia in sterigmatocystin-treated groups. Hyperplasia may also translate DNA damage into harmful genetic mutations, and thus result in oncogene activation or inactivation of tumor suppressor genes, eventually leading to cancer (17).

In Barrett's esophagus, the normal squamous epithelial lining of the esophagus is replaced by columnar cells, thus Barrett's esophagus is presumed to be associated with esophageal adenocarcinoma. Inflammation-associated esophageal tumorigenesis in Barrett's carcinoma (6) was investigated in the reflux esophagitis rat model; however, only hyperplasia of the squamous cells was induced by sterigmatocystin. The involvement of sterigmatocystin in reflux esophagitis and carcinoma thus remains to be determined.

Esophageal squamous epithelial hyperplasia with sterigmatocystin treatment was marginally more evident in the fifth week than in the eleventh week. It is conceivable that the repairing capacity of esophageal squamous cells may be high in rats or the metabolism of sterigmatocystin in rats may be fast. Therefore, sterigmatocystin-induced esophageal cancer genesis may be associated with the amount and duration of exposure to toxic stimuli and esophageal mucosal injury. The doses and duration of treatment with sterigmatocystin that are associated with the development of esophageal cancer have not yet been elucidated. 
A

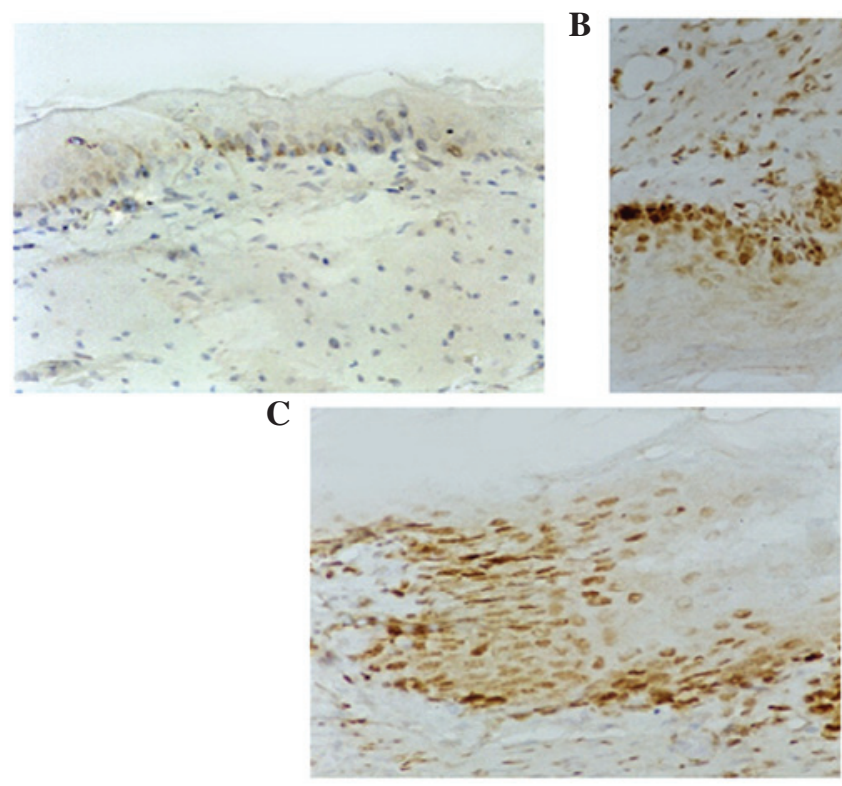

Figure 3. Proliferating cell nuclear antigen (PCNA) immunohistochemical staining (stain, streptavidin-peroxidase, magnification, $\mathrm{x} 400$ ) in (A) control group (B) esophagitis model group and (C) sterigmatocystin-treated group.

A

C
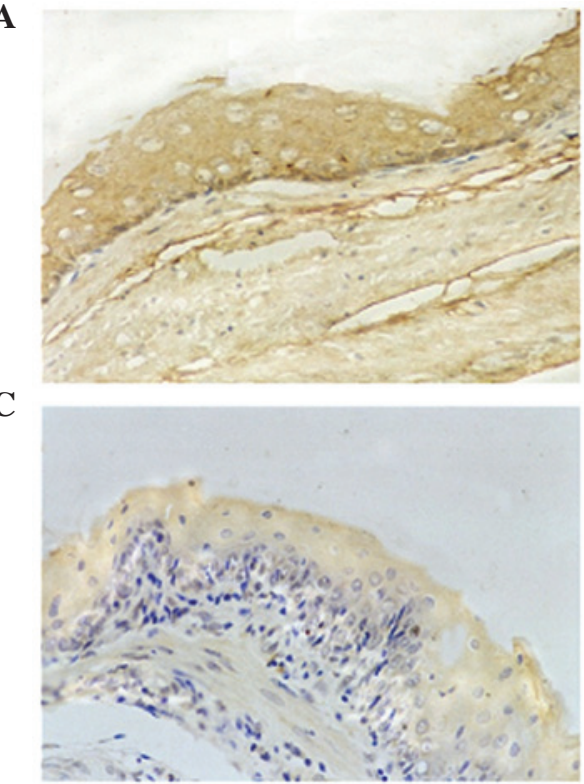

$\mathbf{E}$

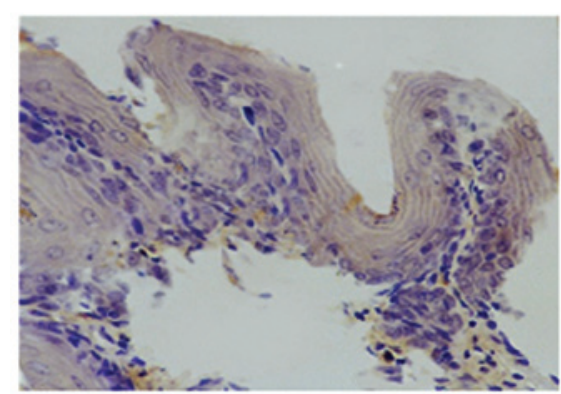

B

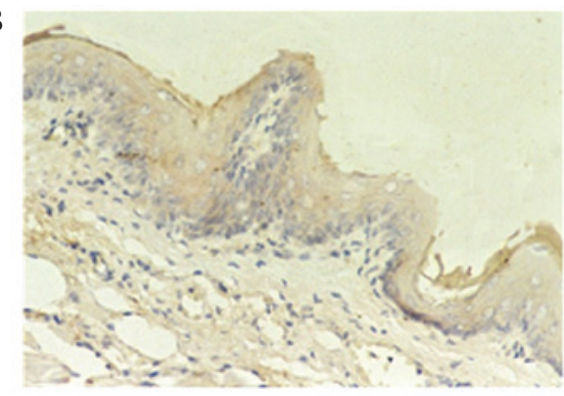

D

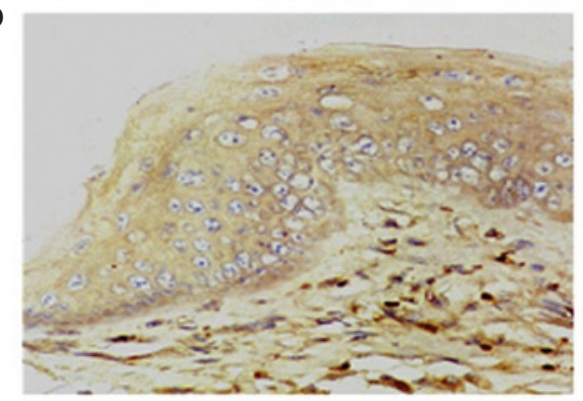

F

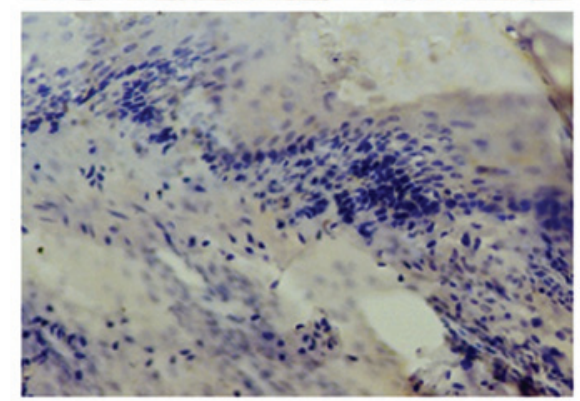

Figure 4. Transporter associated with antigen processing 1 (TAP1) and low molecular weight protein 2 (LMP2) immunohistochemical staining results (stain, streptavidin-peroxidase; magnification, $\mathrm{x} 400$ ) in (A and D) Control group, (B and E) esophagitis model group. (C and F) Sterigmatocystin-treated group. $(\mathrm{A}-\mathrm{C})$ are stained for TAP1 and (D-F) are stained for LMP2.

Furthermore, the results of the present study support the hypothesis that sterigmatocystin negatively impacts the immune function and monocytes $(9,10)$. Sterigmatocystin downregulated the gene expression of TAP1 and LMP2 in human peripheral blood mononuclear cells and inhibited human esophageal squamous cell expression of HLA-I in a 
dose-dependent manner (9,10). Endogenous antigen processing, presentation and the resulting immune disorder are considered to be principal mechanisms of tumor immune escape. The LMP and TAP genes were identified to be involved in the HLA-II gene regions and are considered to be closely associated with the handling of endogenous antigen presentation. Their normal expression is the molecular basis of endogenous antigen-presention $(18,19)$. Endogenous antigens are combined with LMP in the cytoplasm to further cleave peptide fragments. These are transported into the endoplasmic reticulum by TAP, for presentation at the cell surface and identification by circulating T lymphocytes. Thus, LMP and TAP molecular changes may directly affect tumor immunity $(20,21)$, strengthening the ability of a tumor to escape the body's immune surveillance.

Sterigmatocystin exposure resulted in esophageal squamous epithelial cells downregulating the expression of TAP1 and LMP2 in reflux esophagitis rats. Thus, it was suggested that peptide transport is blocked and degradation of endogenous peptides is limited, thus affecting the MHC class I molecules in the endoplasmic reticulum assembly and harming the cell-mediated immune response (19-23). One of the underlying molecular mechanisms of sterigmatocystin-induced esophageal cancer may involve sterigmatocystin functionally altering gene expression and signaling in transformed cells to facilitate their escape from host immune surveillance, thereby promoting cancer onset.

\section{References}

1. Zhang X, Wang F, Wang J, et al: Experimental lung carcinoma induced by fungi and mycotoxins - a review. Beijing Da Xue Xue Bao 35: 4-6, 2003 (In Chinese).

2. Versilovskis A and De Saeger S: Sterigmatocystin: occurrence in foodstuffs and analytical methods - an overview. Mol Nutr Food Res 54: 136-147, 2010

3. Zhang XH and Xue LY: Carcinogenicity and biological effectiveness of sterigmatocystin. Zhonghua Bing Li Xue Za Zhi 38: 136-138, 2009 (In Chinese).

4. Xing LX, Zhang XH, Shen HT, et al: Experimental study on the carcinogenic effects of sterigmatocystin in new born BALB/c mice. Zhonghua Bing Li Xue Za Zhi 36: 265-266, 2007 (In Chinese).

5. Nakajima M, Kato H, Miyazaki T, et al: Tumor immune systems in esophageal cancer with special reference to heat-shock protein 70 and humoral immunity. Anticancer Res 29: 1595-1606, 2009.

6. Abdel-Latif MM, Duggan S, Reynolds JV and Kelleher D: Inflammation and esophageal carcinogenesis. Curr Opin Pharmacol 9: 396-404, 2009.

7. Xu JY, Zhang JK and Wang RM: Control observation on esophageal carcinoma and esophagitis detected by endoscopy between high and low incidence areas of esophageal carcinoma. Chin J Clin Gastroenterol 7: 101-103, 1995 (In Chinese).
8. Ho JA and Durst RA: Detection of fumonisin B1: comparison of flow-injection liposome immunoanalysis with high-performance liquid chromatography. Anal Biochem 312: 7-13, 2003.

9. Huang X, Zhang X, Yan X and Yin G: Effects of sterigmatocystin on interleukin-2 secretion of human peripheral blood mononuclear cells in vitro. Wei Sheng Yan Jiu 31: 112-114, 2002 (In Chinese).

10. Tong P, Zhang X, Yan X, Wang JL and Zhang GJ: Effects of sterigmatocystin on the expression of HLA class I in esophageal squamous cells in vitro. Chin J Exp Surg 23: 583-584, 2006 (In Chinese).

11. Yu ZL: Reflux esophagitis diagnostic criteria and the existing problems. J Chin Med 39: 151-152, 2000 (In Chinese).

12. Fiocca R, Mastracci L, Riddell R, et al: Development of consensus guidelines for the histologic recognition of microscopic esophagitis in patients with gastroesophageal reflux disease: the Esohisto project. Hum Pathol 41: 223-231, 2010.

13. Yuan S and Brookes SJ: Neuronal control of the gastric sling muscle of the guinea pig. J Comp Neurol 412: 669-680, 1999.

14. Miller L, Dai Q, Vegesna A, et al: A missing sphincteric component of the gastro-oesophageal junction in patients with GORD. Neurogastroenterol Motil 21: e813-e852, 2009.

15. Xu Z, Hu T and Liu W: A new procedure in making reliable experimental models of gastroesophageal reflux. Zhongguo Xiu Fu Chong Jian Wai Ke Za Zhi 18: 288-290, 2004 (In Chinese)

16. Rohof WO, Hirsch DP and Boeckxstaens GE: Pathophysiology and management of gastroesophageal reflux disease. Minerva Gastroenterol Dietol 55: 289-300, 2009.

17. Zhu SC, Li R, Wang YX, Feng W, Li J and Qiu R: Impact of simultaneous assay, the PCNA, cyclinD1, and DNA content with specimens before and after preoperative radiotherapy on prognosis of esophageal cancer-possible incorporation into clinical TNM staging system. World J Gastroenterol 11: 3823-3829, 2005.

18. Bandoh N, Ogino T, Katayama A, et al: HLA class I antigen and transporter associated with antigen processing downregulation in metastatic lesions of head and neck squamous cell carcinoma as a marker of poor prognosis. Oncol Rep 23: 933-939, 2010

19. Belicha-Villanueva A, Blickwedehl J, McEvoy S, Golding M, Gollnick SO and Bangia N: What is the role of alternate splicing in antigen presentation by major histocompatibility complex class I molecules? Immunol Res 46: 32-44, 2010.

20. Medina F, Ramos M, Iborra S, de Leon P, Rodriguez-Castro M and Del Val M: Furin-processed antigens targeted to the secretory route elicit functional TAP1-/-CD8+ T lymphocytes in vivo. J Immunol 183: 4639-4647, 2009.

21. Herget M, Kreissig N, Kolbe C, Schölz C, Tampé R and Abele R: Purification and reconstitution of the antigen transport complex TAP: a prerequisite for determination of peptide stoichiometry and ATP hydrolysis. J Biol Chem 284: 33740-33749, 2009.

22. Atkins D, Ferrone S, Schmahl GE, Störkel S and Seliger B: Down-regulation of HLA class I antigen processing molecules: an immune escape mechanism of renal cell carcinoma? J Urol 171: 885-889, 2004

23. Seliger B, Atkins D, Bock M, et al: Characterization of human lymphocyte antigen class I antigen-processing machinery defects in renal cell carcinoma lesions with special emphasis on transporter-associated with antigen-processing down-regulation. Clin Cancer Res 9: 1721-1727, 2003. 